\title{
Simulation des négociations et intercommunalité sur l'étang de Thau
}

Simulation of negotiations on land and urban planning at a territory level. The case of the Thau laguna

\section{Patrick Rio et Sophie Thoyer}

\section{(2) OpenEdition}

\section{Journals}

Édition électronique

URL : http://journals.openedition.org/economierurale/2597

DOI : 10.4000/economierurale.2597

ISSN : 2105-2581

\section{Éditeur}

Société Française d'Économie Rurale (SFER)

Édition imprimée

Date de publication : 5 avril 2010

Pagination : 3-20

ISSN : 0013-0559

\section{Référence électronique}

Patrick Rio et Sophie Thoyer, «Simulation des négociations et intercommunalité sur l'étang de Thau », Économie rurale [En ligne], 316 | mars-avril 2010, mis en ligne le 05 mai 2012, consulté le 06 mai 2019. URL : http://journals.openedition.org/economierurale/2597 ; DOI : 10.4000/ economierurale. 2597 


\section{Simulation des négociations et intercommunalité sur l'étang de Thau}

Patrick RIO • Inra, Laboratoire montpelliérain d'économie théorique et appliquée, Lameta, Montpellier

SOphie THOYER • SupAgro, Lameta, Montpellier

$\mathbf{L}^{\mathrm{c}}$ e mode d'élaboration des politiques publiques évolue pour privilégier des démarches locales de concertation et de contractualisation. En France, les démarches de planification concertée se sont imposées avec force sur les questions d'aménagement du territoire et de gestion de l'eau (Barraque, 1997).

Parallèlement, la loi relative au renforcement et à la simplification de la coopération intercommunale de 1999 (dite loi Chevènement) a simplifié le paysage des collectivités locales et a donné la possibilité aux Établissements publics de coopération intercommunale (EPCI) de prendre en charge, entre autres, les compétences d'assainissement et d'environnement sur leur territoire. La loi Solidarités et renouvellement urbain (SRU) de 2000 a aussi modernisé les documents d'urbanisme en instituant le Schéma de cohérence territoriale (SCOT) qui doit assurer une meilleure cohérence entre les politiques publiques élaborées à l'échelle d'une agglomération.

La mise en place des Schémas d'aménagement et de gestion des eaux (SAGE), lancés dans le cadre de la loi sur l'eau de 1992, a progressivement imposé la pratique de gestion concertée à l'échelle des bassins versants. Cette tendance s'est accentuée avec la Directive cadre sur l'Eau de 2000, directement inspirée par le système français. Mais on trouve aussi d'autres déclinaisons, moins encadrées institutionnellement, comme la gestion intégrée des zones côtières ou les schémas de mise en valeur de la mer. Dans les Commissions locales de l'eau (CLE), au sein desquelles s'élaborent les projets de SAGE, les collectivités territoriales, et les communes en particulier, sont présentes et leur engagement dans la concertation a tendance à se renforcer (Ferroudji-Richard, 2008) : elles ont compris l'intérêt de coordonner non seulement leurs projets d'investissement concernant les aménagements et les équipements relatifs à l'eau mais aussi leurs objectifs de qualité environnementale et de gestion de la ressource en eau. De fait, la loi sur l'eau et les milieux aquatiques (LEMA) de décembre 2006 impose que les décisions prises dans les SAGE soient respectées par les documents de planification territoriale tels les SCOTs.

Les animateurs des structures de négociation sont relativement démunis lorsqu'il s'agit de décider comment organiser la structuration de la négociation. Leur tâche est compliquée par la construction des intercommunalités, car la délégation aux intercommunalités des compétences traditionnellement assurées au niveau des communes peut bouleverser la structure des préférences qui s'expriment à la table des négociations et faire évoluer la nature des compromis obtenus. Comme un processus de négociation ne peut être « rejoué », l'économiste se doit de proposer des outils de simulation permettant à l'animateur de mieux anticiper l'impact des configurations de la négociation sur les accords.

Cet article développe un outil de simulation qui permet de prendre en compte deux questions spécifiques : évaluer les préférences des habitants sur la politique de gestion d'un territoire (en l'occurrence un bassin-versant) et identifier les conséquences d'une politique négociée à l'échelle des EPCI plutôt qu'à l'échelle des communes, ou à une échelle intermédiaire. 
Notre méthodologie est basée sur l'utilisation d'un modèle de marchandage multilatéral couplée à une méthode originale de révélation des préférences des acteurs d'un territoire, fondée sur la théorie politiciensélecteurs et mobilisant une analyse économétrique de l'électorat communal. Elle a l'avantage d'être relativement simple à mettre en œuvre et de permettre de simuler très rapidement l'impact de différentes structures de négociation (voir encadré concernant le choix de la méthodologie).

Nous illustrons la faisabilité et l'intérêt de cette méthodologie en l'appliquant au cas de la gestion du bassin de Thau, situé en bordure de la Méditerranée au sud-ouest de Montpellier. C'est un site à vocation multiple : bassin conchylicole, destination touristique et sportive, et élément remarquable du paysage, il est inséré dans un bassin-versant à vocation viticole et affecté par la croissance urbaine forte de Montpellier et Sète. La lagune de Thau a fait l'objet depuis la fin des années 1980 de nombreux documents de planification et de gestion pour maintenir la qualité de ses eaux, menacée par les rejets urbains, la pollution diffuse d'origine agricole et la sur-fréquentation, et tenter d'équilibrer les différents usages qui en sont faits. Mais ces efforts collectifs, formalisés dans le premier contrat de baie signé en 1990 et dans le Schéma de mise en valeur de la mer (SMVM) approuvé en 1995, n'ont pas eu l'impact escompté, en particulier en raison de la difficulté à construire des préférences collectives stables pour les 21 communes du bassin-versant, les communes littorales étant résolument tournées vers le tourisme et la vocation halieutique de la lagune, tandis que les communes rurales sont plus préoccupées de l'avenir viticole de la région. Depuis 2000, ces communes se sont organisées en trois EPCI (deux communautés d'agglomération et une communauté de commune). La négociation sur un Schéma d'aménagement et de gestion des eaux a été engagée, simultané- ment avec la négociation sur un schéma de cohérence territoriale, toutes deux portées par la même structure gestionnaire, le Syndicat mixte du bassin de Thau (SMBT).

Nous montrons dans cet article comment la structuration de la négociation en fonction de la géométrie des intercommunalités fait évoluer les priorités de gestion du bassinversant et les compromis obtenus. Cette analyse, au-delà de l'éclairage fourni sur le cas du bassin de Thau à la veille de l'ouverture des négociations pour la mise en place d'un SCOT et d'un SAGE, permet d'illustrer comment on peut construire un outil de simulation des négociations facile à mettre en œuvre et illustrant les difficultés inhérentes au regroupement intercommunal.

La première partie de cette étude présente le cadre méthodologique utilisé. Son application au cas du bassin de Thau est exposée en deuxième partie suivie, en troisième partie, de commentaires sur les résultats obtenus et des développements ultérieurs possibles. La conclusion permettra d'offrir des éléments de réflexion intéressants, utiles aux autorités publiques.

\section{Comment modéliser et simuler les démarches de négociation?}

Le rôle de la concertation dans l'élaboration des politiques publiques est régulièrement analysé par la sociologie et les sciences politiques (Beuret, 2006 ; Claeys-Mekdade, 2003). L'économie standard, en revanche, restreint son analyse à une interprétation de la concertation comme une négociation dans laquelle les intérêts de plusieurs parties prenantes se confrontent pour trouver un compromis qui puisse améliorer au moins la situation de l'un sans détériorer celle des autres. Elle s'appuie donc le plus souvent sur des modèles de théorie des jeux coopératifs ou non coopératifs (voir Dinar et al., 2008, pour une revue récente), qui font certes des hypothèses simplificatrices sur le déroulement d'une négociation et sur la représen- 
tation des préférences des agents, mais qui permettent en revanche de fournir un cadre conceptuel riche pour modéliser et analyser les relations entre la structuration de la négociation (structure informationnelle, structure de coalition, hiérarchie des préférences, règles de décision), les stratégies des parties prenantes et les équilibres obtenus.

Notre objectif étant de fournir un modèle de simulation qui puisse être appliqué à différents cas de négociation territoriale, nous avons choisi de nous appuyer sur une représentation simplifiée qui ne prétend pas capturer toutes les dimensions d'une vraie concertation. Les aspects psychologiques, la dynamique du groupe, par exemple, ne sont considérés qu'au travers d'un paramètre mesurant l'influence relative de chaque agent. Ce choix nous permet de nous concentrer sur les enjeux liés à la structuration de la négociation et à l'organisation des parties prenantes.

\section{Le modèle de marchandage}

Nous modélisons la démarche de concertation lors de l'élaboration collective d'un document de planification comme un modèle de négociation, dérivé de la théorie des jeux non coopérative. Certes, ce choix repose sur plusieurs hypothèses simplificatrices.

- L'espace de négociation est clairement défini : autrement dit, les enjeux à négocier (les variables de négociation) sont identifiés, bornés (par exemple par les obligations réglementaires) et en nombre fini. La dynamique de la négociation ne permet pas l'introduction de nouveaux paramètres, l'identification des variables à négocier relevant d'une étape antérieure du processus. Dans les procédures d'élaboration de SAGE ou de SCOT, c'est souvent la phase de diagnostic qui joue ce rôle d'identification et de stabilisation des variables à négocier.

- On peut représenter les préférences des parties prenantes de la négociation par des fonctions d'utilité qui dépendent de la valeur des variables négociées. Les fonctions d'utilité des agents ne varient pas au cours de la négociation. Cette hypothèse est cohérente avec la précédente (espace de négociation bien défini) et les hypothèses informationnelles suivantes. - Les parties prenantes agissent en information parfaite et complète : chaque joueur connaît la structure de préférences des autres parties prenantes, les règles de négociation et le poids politique de chacun dans la négociation ${ }^{1}$. De fait, les collectivités locales, qui ont des occasions multiples de débattre et donc de se connaître, sont effectivement plutôt bien informées sur les enjeux que la négociation représente pour chacune d'entre elles et sur leurs poids politiques.

- Si les parties prenantes n'arrivent pas à se mettre d'accord sur une solution commune, c'est une partie tiers (l'État par exemple) qui prendra une décision et cette décision risque d'être plus défavorable aux parties prenantes que n'importe lequel des accords qui aurait pu être obtenu. C'est cette condition qui assure que les parties prenantes rentreront dans le processus de négociation. On la retrouve assez fréquemment dans la décision publique (par exemple cofinancement de l'État si un accord local est atteint).

À partir de ces hypothèses, nous construisons une structure de marchandage multilatéral, sur la base du modèle proposé par Rausser et Simon (1991), Adams et al. (1996), et Thoyer et al. (2001), permettant de simuler la négociation entre plusieurs parties prenantes sur plusieurs variables négociées simultanément. Les résultats numériques permettent de fournir deux types d'information :

- évaluer comment se déforme le compromis obtenu sur les variables négociées selon la structure de négociation choisie ;

- évaluer la manière dont les efforts (les renoncements) se répartissent entre les parties prenantes.

1. D'autres versions du modèle, plus sophistiquées, permettent de relâcher cette hypothèse (Quérou et al., 2006). 
La négociation s'organise entre $N$ agents, par exemple des communes ou des EPCI, négociant sur $m$ politiques communes à mettre en œuvre, par exemple le taux d'urbanisation ou les investissements à prévoir pour l'assainissement collectif. Chaque agent $i$ a un point idéal $A^{i}$ qui est la solution qu'il choisirait s'il n'avait pas à négocier de compromis avec d'autres agents.

$A^{i}$ est donc un vecteur à $m$ dimensions $A^{i}$ $=\left(\mathrm{A}_{1}^{\mathrm{i}}\right)_{\mathrm{l}=1: \mathrm{m}}$. Chaque agent est aussi caractérisé parce qu'on appellera une fonction de désutilité $D U_{i}$. Cette fonction mesure la désutilité que cet agent éprouve lorsqu'il s'éloigne de sa position idéale ${ }^{2} A^{i}$. La négociation doit permettre d'aboutir à une décision collective unique portant sur la valeur des $m$ politiques à mettre en œuvre. On la décrit par un vecteur à $m$ éléments $X=\left\{X_{1}\right\}_{1=1: \mathrm{m}}$

Nous choisissons de représenter la désutilité de l'agent $i$ liée à l'adoption de $X$ par une fonction de distance $D U$ (la désutilité augmente lorsqu' on s'éloigne de la position idéale).

La désutilité de l'agent $i \mathrm{~s}$ 'exprime donc :

$D U_{i}=\sqrt{\sum_{l=1}^{m} \gamma_{i}^{i}\left(A_{i}^{i}-X_{l}\right)^{2}}$

avec $A_{l}^{i}$ le point idéal de l'agent $i$ pour la politique $l$

$\gamma_{l}^{i}$ l'intensité de la préférence de l'agent $i$ pour la politique $l$

2. Les points idéaux des joueurs pris séparément n'assurent pas forcément un optimum social. De fait, l'absence de coordination peut aboutir à une dégradation des ressources dommageable pour tous. Certes, la négociation a pour principal objectif d'aboutir à une position commune génératrice de bien public (amélioration de l'environnement par exemple) et donc meilleure pour l'intérêt collectif mais nous faisons l'hypothèse que ces avantages seront partagés par tous. Nous nous focalisons donc ici sur le partage des efforts à fournir. Ceci explique que nous traduisions les effets de la négociation en termes de désutilité.
La dynamique de la négociation consiste à alterner une phase de propositions durant laquelle chaque agent $\mathrm{i}$ fait une proposition $X_{i}$ sur les $m$ politiques négociées, et une phase de décision durant laquelle les agents choisissent d'accepter ou de rejeter les propositions faites. La négociation s'arrête si tous les joueurs s'accordent sur une proposition, qui est alors mise en œuvre. Si on applique une règle d'unanimité, il suffit qu'un joueur rejette la proposition en cours pour qu'un nouveau tour soit engagé, dans lequel chaque agent $i$ est amenée à émettre une nouvelle proposition avec une probabilité $w_{i}$. Cette probabilité pourrait s'interpréter comme l'influence relative du joueur $i$ à la table des négociations : un joueur plus puissant, ou plus haut placé, voire plus éloquent, a plus de chance de faire valoir ses propositions dans la négociation. De manière équivalente, on peut considérer l'étape de proposition comme un moment où, chaque joueur i ayant émis (de manière simultanée avec les autres joueurs) une proposition $X^{i}$, une de ces $N$ propositions $X^{i}$ sera sélectionnée avec la probabilité $w_{i}$, pour être ensuite proposée au vote du groupe. L'équivalence assure la possibilité pour chaque joueur $j$ de calculer l'espérance de perte ${ }^{3}$ qu'il encourt en choisissant de rejeter la proposition faite pour passer au tour suivant $k$.

Son espérance de perte s'écrit :

$E_{k}\left(D U_{j}\right)=\sum_{i=1}^{N} w_{i} D U_{j}\left(X_{k-1}^{(i)}\right)$.

Avec :

$D U_{j}\left(X_{k-1}^{(i)}\right)$ : désutilité de l'agent $j$ associée à la proposition de l'agent $i$ au tour $k-1$.

3. Une interprétation possible de cette désutilité espérée serait en terme de "désutilité de réservation » ou de « point de retrait » tel que, s'il n'est pas satisfait, le joueur se retire du marchandage (et l'accord ne peut avoir lieu). 
RECHERCHES

Patrick RIO, Sophie THOYER

La négociation s'arrête lorsque tous les joueurs présents choisissent d'accepter la proposition en cours. Formellement, le critère d'arrêt de la négociation est le suivant :

il n'existe pas de proposition $X_{k}^{(i)}$ telle que pour au moins un joueur $j$ ( $i$ compris), la désutilité de cette proposition soit inférieure à la désutilité espérée $E_{k}\left(D U_{j}\right)$.

De fait, la dynamique de la négociation est donc simple : chaque joueur sait qu'en cas de refus de participation, la solution qui serait retenue par le régulateur pourrait être la plus mauvaise pour lui. Chaque joueur propose donc au premier tour la solution la plus favorable pour lui-même, souvent défavorable aux autres. Chaque proposition pouvant être retenue comme solution avec une probabilité $w$, chaque joueur a intérêt à rechercher une proposition qui soit meilleure pour lui-même que ce qu'il peut espérer si le jeu s'arrête à ce tour, tout en respectant les gains que les autres joueurs peuvent espérer si le jeu s'arrête à ce tour. La proposition respectant nécessairement les gains espérés des autres parties amène un gain à chaque tour moins favorable au joueur qui l'énonce. Mais chaque joueur concédant quelque chose aux autres à chaque proposition, l'espérance de gain à chaque tour est plus favorable aux joueurs. Le jeu de propositions simultanées entraînant une suite décroissante des gains mais aussi une suite croissante d'espérance de gain, la dynamique du marchandage sera convergente dans les gains : il arrivera un tour où les gains de la proposition de chacun ne seront plus distinguables des gains espérés compte tenu des propositions des autres joueurs. Le compromis sera atteint.

Sous des conditions assez générales de compacité de l'espace de négociation et de concavité des fonctions d'utilité des joueurs, on montre que cette dynamique converge vers un point limite atteignable en temps fini (Quérou et al., op. cit.). Le mécanisme de résolution est illustré dans l'annexe 1.

\section{Révélation des préférences des parties prenantes}

Dans notre modélisation, nous nous focalisons sur les négociations entre communes ou EPCI. Chaque partie prenante, commune ou communauté de communes ou d'agglomération, doit être caractérisée par une fonction de désutilité $D U_{i}$ qui s'analyse comme la perte d'utilité agrégée des électeurs de cette circonscription résultant de l'acceptation d'un compromis avec les objectifs des électeurs des autres circonscriptions.

Par rapport aux outils existants (le MultiAttribute Value Theory de Keeney et Raiffa, 1976 ; le Multi Criteria Decision Analysis de Mustajoki et al., 2004 ; le Analytic Hierarchy Process de Saaty, 1990), notre approche vise surtout à la simplicité en conservant une interprétation rigoureuse de l'action publique. Précisément, elle n'exige que le traitement de statistiques déjà disponibles pour situer les positions relatives des communes.

Cette information suffit pour estimer la structure des préférences des parties prenantes c'est-à-dire, dans notre exemple de l'étang de Thau, l'évaluation des paramètres $\gamma_{l}^{i}$ et $\mathrm{A}_{l}^{i}$ des fonctions de désutilité des communes (voir équation 1). Cette facilité résulte des apports de la théorie des choix publics, basée sur une inversion des fonctions de préférence politique.

La théorie des choix publics se refuse à considérer le décideur public comme un dictateur bienveillant au service de l'intérêt général. Elle fait l'hypothèse que ce décideur cherche à mettre en place des politiques publiques $p_{j}$ qui favorisent les groupes d'intérêt qui lui ont fourni (ou lui promettent) le soutien politique le plus important. La fonction d'utilité $U$ du décideur (aussi appelée sa fonction de préférence politique) est la somme pondérée du bien être des groupes d'intérêts i composant son électorat, $W_{i}\left(p_{j}\right)$, engendré par les politiques $p_{j}$.

$U=\sum \alpha_{i} W_{i}\left(p_{j}\right)$ 
La pondération $\alpha_{i}$ reflète la préférence relative du décideur pour les différents groupes. Si on connaît la manière dont le bien-être des groupes varie avec le niveau des politiques $p_{j}$, on peut, par l'observation des politiques mises en œuvre et en faisant l'hypothèse que le décideur maximise sa fonction de préférence politique $U$, révéler les poids $\alpha_{i}$ qu'il donne aux différents groupes.

Du point de vue de la préférence politique, ce résultat est obtenu pour les conditions de premier ordre :

$$
\frac{\partial U}{\partial p_{j}}=\sum_{i} \alpha_{i} \times \frac{\partial W_{i}}{\partial p_{j}}=0
$$

La théorie du choix public invite alors à calculer $\frac{\partial W_{i}}{\partial p_{j}}$, permettant ainsi de révéler les poids politiques $\alpha_{i}$ de chaque groupe d'intérêt i dans la fonction de préférence politique du gouvernement.

Nous inversons cette approche : en faisant l'hypothèse que le conseil municipal élu a une fonction de préférence politique qui est la somme pondérée de la satisfaction des différentes composantes socio-économiques de son électorat. La pondération se fait en fonction de la puissance électorale de chaque catégorie socio-économique, c'est-à-dire en fonction simplement de leur poids numérique dans la commune. Nous connaissons donc $\alpha_{i}$, le poids politique de chaque catégorie sociale $i$. Reste à estimer $W_{i}\left(p_{j}\right)$, la préférence de la catégorie sociale i relative à la politique $p_{j}$. Ainsi l'hypothèse est donc faite que les préférences sur les politiques de la commune sont fortement corrélées aux catégories socioprofessionnelles. Nous posons la régression suivante :

$p_{j}=\sum_{i} \beta_{i, j} X_{i}+\sum_{k} \beta_{k, j} Z_{k}+u_{i}$

où :

$-p_{j}$ est la variable mesurant les décisions passées de la commune sur le niveau de la $j^{\text {ème }}$ politique,

- les $X_{i}$ sont les parts relatives des catégories socio-professionnelles $i$ dans la population totale de la commune (mesurées par les statistiques INSEE). Par analogie, $X_{i}$ qui est une variable dans l'équation (3) est équivalent au $\alpha_{i}$ de l'équation (2),

- les $Z_{k}$ sont des variables exogènes susceptibles d'affecter mécaniquement les politiques $p_{j}$,

$-u_{j}$ est un terme d'erreur, suivant une loi normale centrée $N(0, \sigma)$.

Deux ensembles de paramètres sont alors à estimer, les $\beta_{k, j}^{\wedge}$ dont l'intérêt technique est peu pertinent pour notre analyse et les $\beta_{i, j}^{\wedge}$ qui sont les préférences révélées des différentes composantes sociales pour la politique $p_{i}$. C'est le facteur $W_{i}\left(p_{j}\right)$ de l'équation (2).

Comme $\beta_{i, j}^{\wedge}$ peut prendre une valeur négative, nous procédons à la normalisation suivante :

\begin{tabular}{|c|c|c|}
\hline Condition & Point idéal & Intensité \\
\hline$\beta_{i, j}^{\wedge}<0$ & $\mathrm{~A}_{\mathrm{i}, \mathrm{j}}=0$ & $\pi_{\mathrm{j}}=\left|\sum_{i} \beta_{i, j}^{\wedge} X_{i}\right|$ \\
\hline$\beta_{i, j}^{\wedge} \geq 0$ & $\mathrm{~A}_{\mathrm{i}, \mathrm{j}}=1$ & $\pi_{\mathrm{j}}=\left|\sum_{i} \beta_{i, j} X_{i}\right|$ \\
\hline
\end{tabular}

On retient ainsi une même mesure de l'intensité d'un choix de politique en prenant la valeur absolue de cette orientation mais on la fait s'exercer positivement ou négativement en associant une cible nulle ou unitaire, selon que cette intensité s'exerce à l'encontre de cette orientation ou en sa faveur.

\section{Les scenarii du marchandage}

Ayant précisé la manière d'obtenir les paramètres de la fonction de désutilité de chaque commune, nous pouvons nous concentrer sur les simulations qu'un gestionnaire serait intéressé à mettre en œuvre. Dans notre contexte institutionnel, il cherchera vraisemblablement à obtenir un accord entre les établissements publics de coopération intercommunale (communauté de communes ou d'agglomération). Il s'agit d'une démarche a priori efficiente dans la mesure où l'agrégation préalable des préférences des communes réduit le nombre des inter- 
locuteurs et facilite une exploration plus approfondie des choix proposés. L'efficience dépend cependant des conditions dans lesquelles ces EPCI prennent en charge les positions dispersées de leurs communes membres.

L'originalité de notre instrument consiste justement à évaluer cet effet d'agrégation. Pour cela, nous confrontons deux situations polaires : l'accord décentralisé obtenu d'une négociation directe entre les communes et l'accord centralisé obtenu d'une négociation directe entre EPCI. Entre ces deux pôles, nous introduisons différentes configurations permettant de saisir l'effet de divers moyens de pression dont les communes disposent à l'égard des EPCI pour mieux faire respecter leurs préférences. Les configurations intéressantes sont évidemment celles qui peuvent être traduites dans la pratique par des règles de décision fixées aux négociateurs. Par exemple, nous supposerons l'existence d'une négociation préalable entre les membres de chaque EPCI dont l'utilisation suivra l'une des deux règles suivantes : (1) chaque EPCI prendra pour point idéal l'accord obtenu entre les communes membres - ce cas sera dit «PI-contrainte»; ou (2) la désutilité associée à l'accord entre communes-membres de l'EPCI sera une borne explicite pour l'EPCI et constituera son point de retrait : toute proposition qui aboutirait à une désutilité supérieure à cette désutilité plafond, sera rejetée par l'EPCI dans la négociation avec les autres EPCI. $\mathrm{Ce}$ cas sera dit «PM-contrainte » car elle correspond à une contrainte de participation par point de menace. Il faut noter que ce choix laisse place à plus d'arrangement dans l'accord terminal, car seule la désutilité globale - qui permet des compensations entre les différentes dimensions de la politique - est considérée.

Notre approche fournit au gestionnaire un jeu de références qui lui permettront d'identifier les issues des négociations et leur robustesse lorsque varient les configu- rations de la négociation. Il reste à proposer une mesure de cette robustesse.

On peut mesurer cette robustesse en évaluant ce que concèdent les communes dans une structure de négociation déterminée, c'est-à-dire la désutilité qu'elles subissent du fait de l'accord obtenu. Des notions de désutilité maximale au sein d'un groupe de commune, de désutilité moyenne pour le groupe ou encore de variance de cette désutilité sont des indicateurs synthétiques des tensions associées à l'accord issu d'une structure particulière de la négociation. Une variance faible témoigne d'une redistribution modérée des pertes, une variance forte indiquera l'existence d'acteurs fortement frustrés par l'accord. Notons que ces estimateurs ont une transcription graphique immédiate qui facilite l'identification des structures préférables.

\section{La modélisation du bassin de Thau}

\section{Le contexte de la négociation}

Les 7500 hectares de la lagune de Thau en font l'une des plus importantes de France. Les activités littorales sont vouées essentiellement à l'activité industrialo-portuaire (avec le port de Sète), conchylicole (huîtres, moules et pêche de coquillages) et au tourisme, avec un poids grandissant mis sur les activités nautiques de loisir. L'occupation du bassin-versant se partage entre terrains viticoles et zones urbaines en forte croissance, traversées d'infrastructures routières et ferroviaires importantes. La principale caractéristique de l'étang de Thau est la multiplication des usages du site et de l'eau de la lagune, et la fréquence de conflits liés au partage de ces ressources (Rey-Valette, Valette ; 2005). À partir du milieu des années 80 , la gestion de la lagune de Thau, qui avait été jusque-là surtout le fait de politiques sectorielles cloisonnées, va, sous l'impulsion de l'État, se tourner vers des objectifs plus transversaux, mieux calés sur les dynamiques des territoires du bassinversant. L'un des éléments déclencheurs a 
été la crise majeure d'eutrophisation de la lagune en 1989 qui a induit une catastrophe économique pour les conchyliculteurs. On reconnaît alors la nécessité d'articuler gestion environnementale du bassin-versant et mesures de préservation de la qualité de l'eau dans la lagune. Des contrats de baie successifs (1990-1995 ; 1996-2002 ; 20042008) renforcés par la signature en avril 1995 par le préfet du Schéma de mise en valeur de la mer adopté par les 9 communes limitrophes de l'étang serviront à conforter la vocation conchylicole de la lagune. Le partenariat entre l'État et les collectivités locales concernées se renforce donc à travers une démarche contractuelle négociée.

Les 21 communes $^{4}$ du bassin-versant de l'étang de Thau ont des vocations et des priorités différentes. Il est clair que les communes littorales comme Bouzigues, Mèze, Marseillan, s'appuient sur une activité dominante tournée vers la pêche et la conchyliculture, avec des élus locaux très proches de ces milieux professionnels. D'autres communes sont plus sensibles au développement du tourisme ou à l'accueil des ménages retraités comme Balaruc ou Frontignan. Les petites communes rurales (Loupian, Pinet, Pomerols), éloignées du littoral, se sentent souvent loin des préoccupations de la lagune et ont parfois du mal à accepter que toutes les orientations du bassin soient tournées vers la qualité des eaux, au détriment de leur capacité à investir dans leurs activités traditionnelles que sont la viticulture et dans une moindre mesure, le tourisme rural. Enfin, certaines communes comme Gigean ou Fabrègues, bien que dans le bassin-versant de Thau, sont entièrement tournées vers la communauté d'agglomération de Montpellier. Elles sont soumises à une pression démographique forte, s'orien-

4. Agde, Aumelas, Balaruc les Bains, Balaruc le Vieux, Bouzigues, Castelnau de Guers, Cournonsec, Fabrègues, Florensac, Frontignan, Gigean, Loupian, Marseillan, Mèze, Montagnac, Montbazin, Pinet, Pomerols, Poussan, Sète, Villeveyrac. tant vers l'accueil des ménages travaillant sur Montpellier.

Depuis 2000, ces communes se sont structurées dans différents EPCI (annexe 2). Trois EPCI sont directement concernés par l'avenir de la lagune et participent, à des degrés divers, aux différentes instances de concertation sur la planification des activités ayant un impact sur Thau. Elles ont toutes en commun d'avoir choisi de partager la compétence « protection et mise en valeur de l'environnement ». La Communauté d'agglomération du bassin de Thau (CABT) regroupe 8 communes à l'est du bassin, dont 6 ont l'intégralité de leur territoire dans le bassin-versant de Thau. Elle est très mobilisée par la question de la protection de la lagune car une grande partie de ses activités économiques en dépend. La communauté d'agglomération Hérault-Méditerranée (CAHM) regroupe 13 communes à l'ouest de la lagune, dont 6 sont dans le périmètre du bassin-versant. En dehors de la ville d'Agde, il s'agit de petites communes rurales, plutôt viticoles et relativement éloignées des activités de la lagune, même si elles bénéficient de son image. La communauté de communes du nord du bassin de Thau (CCNBT) ne regroupe que 6 communes mais elles sont toutes situées dans le bassin-versant. Trois communes sont rattachées à des EPCI plus éloignés : Fabrègues et Cournonsec appartiennent à la communauté d'agglomération de Montpellier, Aumelas appartient à la communauté de communes Vallée de l'Hérault. Dans la suite de l'analyse, elles sont considérées comme un groupe à part, hors communauté du bassin (HCB).

Il n'est pas anodin de signaler qu'un projet de fusion entre la communauté d'agglomération du bassin de Thau et la communauté de communes du nord du bassin de Thau a existé, même s'il est aujourd'hui au point mort, probablement pour des raisons de lutte d'influence et de stratégie politique. En revanche, ces deux EPCI ont créé un syndicat mixte (Syndicat mixte du bassin de Thau, 
RECHERCHES

Patrick RIO, Sophie THOYER

Tableau 1. Les coefficients de préférence en fonction du groupe social

\begin{tabular}{l|c|c|c|c}
\hline $\begin{array}{c}\text { Coefficient de préférence } \\
\text { Groupe social }\end{array}$ & $\begin{array}{c}\text { Croissance } \\
\text { urbaine }\end{array}$ & $\begin{array}{c}\text { Croissance } \\
\text { touristique }\end{array}$ & Équipement & Assainissement \\
\hline Agriculteur & 1,0536 & $-0,5905$ & 0,2823 & $-0,1214$ \\
\hline Artisan & 0,1990 & 0,1515 & 0,9995 & 0,5467 \\
\hline Cadre & 0,6949 & 0,1971 & $-0,8609$ & $-0,5482$ \\
\hline Prof,-Intermédiaire & $-1,3435$ & $-0,6088$ & 1,4203 & 0,3059 \\
\hline Employé & $-0,0042$ & $-1,2672$ & 0,3826 & $-0,4158$ \\
\hline Ouvrier & $-0,3857$ & $-0,0298$ & 0,8498 & 0,9014 \\
\hline Retraité & $-1,5747$ & 0,4570 & 0,4756 & $-0,1162$ \\
\hline Autre - sans activité & 1,0663 & 1,3455 & $-1,5125$ & $-0,4442$ \\
\hline Mas (conchyliculteurs) & $-0,2794$ & $-0,1611$ & $-0,1048$ & 0,6580 \\
\hline
\end{tabular}

Source : estimations des auteurs

SMBT) en 2005, chargé de piloter plusieurs outils : un contrat qualité de la lagune de Thau, le SCOT du bassin de Thau, et le Schéma d'aménagement et de gestion des eaux du bassin de Thau. C'est dans cette perspective que doivent se négocier entre les EPCI concernés un certain nombre de politiques clé, qui affectent simultanément la qualité environnementale de la lagune et les choix de développement économique.

Pour simplifier, nous avons considéré quatre éléments clé de la politique d'aménagement et de protection de l'environnement, qui sont négociés au sein de chaque EPCI mais aussi entre EPCI pour favoriser une gestion cohérente du bassin-versant : - le taux de croissance urbaine : cela dépend évidemment des choix faits sur les plans locaux d'urbanisme, en cohérence avec le SCOT (données Insee RPG, 19995), - le taux de croissance des activités touristiques : nous avons intégré dans cet indicateur le nombre de places de camping, d'hôtellerie, le ratio des résidences secondaires par rapport aux résidences principales (CRT LR, $2002^{6}$; Insee, 1999),

5. Insee (1999). Recensement Général de la Population. http://www.recensement-1999.insee.fr/ RP99/rp99/page_accueil.paccueil

6. Comité Régional du Tourisme - Languedoc Roussillon (2002). « Les chiffres du camping 19901998-2000- Fédération de l'hôtellerie de plein air ». http://www.languedoc-roussillon.prefgouv.fr/ actions/missionlittoral/ref_bibliographiques.shtm
- le taux d'équipement institutionnel et commercial des communes qui est un indicateur du confort de vie des résidents (à partir des données Insee, op. cit.),

- les efforts à fournir en matière d'assainissement. Il s'agit de moderniser et d'agrandir la capacité des stations d'épuration existantes et de mieux gérer les effluents des habitats ou activités non raccordées au réseau. Les données sont celles de la capacité nominale de traitement en équivalent habitant (Agence de l'eau RMC).

\section{Résultats sur les préférences des communes}

Les préférences de chacune des 21 communes ont été calculées à partir d'une estimation (par la méthode inversée de révélation des préférences expliquée plus haut) des coefficients de préférence de neuf catégories socio-économiques à l'égard des quatre dimensions de la négociation : croissance urbaine, croissance touristique, équipement, assainissement. Les variables exogènes $Z_{k}$ sont la tendance à long terme de l'urbanisation (qui capte l'intensité de la demande résidentielle du fait de l'urbanisation en place), le revenu imposable, le taux de chômage, les mouvements pendulaires quotidiens rapportés à la population active.

Les coefficients de préférence obtenus sur l'ensemble du bassin-versant sont rapportés au tableau 1. 
Tableau 2. Point idéal et intensité des préférences des EPCI

\begin{tabular}{l|c|c|c|c}
\multicolumn{1}{c|}{ Point idéal des EPCI } & $\begin{array}{c}\text { Croissance } \\
\text { urbaine }\end{array}$ & $\begin{array}{c}\text { Croissance } \\
\text { touristique }\end{array}$ & Équipement & Assainissement \\
\hline HCB & 1 & 0 & 1 & 0 \\
\hline CABT & 0 & 1 & 0 & 1 \\
\hline CCNBT & 0 & 0 & 0 & 1 \\
\hline CAHM & 0 & 1 & 0 & 0 \\
\hline $\begin{array}{l}\text { Intensité des préférences des } \\
\text { communautés d'agglomération }\end{array}$ & $\begin{array}{c}\text { Croissance } \\
\text { urbaine }\end{array}$ & $\begin{array}{c}\text { Croissance } \\
\text { touristique }\end{array}$ & Équipement & Assainissement \\
\hline HCB & 0,4360 & 0,1317 & 0,3823 & 0,0499 \\
\hline CABT & 0,2072 & 0,3174 & 0,3188 & 0,1566 \\
\hline CCNBT & 0,2241 & 0,1946 & 0,3153 & 0,2660 \\
\hline CAHM & 0,1657 & 0,3776 & 0,3282 & 0,1285 \\
\hline
\end{tabular}

HCB hors communauté du bassin ; CABT communauté d'agglomération du bassin de Thau ; CCNBT communauté de communes du Nord du bassin de Thau ; CAHM communauté d'agglomération Hérault-Méditerranée.

Source : calculs des auteurs

Cette analyse fait donc l'hypothèse implicite que les catégories socioprofessionnelles ont des préférences relativement homogènes, indépendantes de leur choix de localisation. Autrement dit, un retraité d'Aumelas dans l'arrière-pays a les mêmes préférences qu'un retraité de Balaruc-lesBains sur les rives de l'étang de Thau. Mais on constatera que la proportion de retraités est plus importante à Balaruc, traduisant leur « vote avec les pieds ", c'est-à-dire leur tendance à s'installer là où la politique locale défendue correspond mieux à leurs attentes.

Comme attendu, les intérêts et les positions des agents à l'égard de ces diverses politiques sont très variables. L'interprétation précise de ces positions demanderait une connaissance approfondie du terrain. On notera par exemple que la politique d'assainissement a pour principaux soutiens les conchyliculteurs, les ouvriers (dont l'emploi dépend en grande partie de l'activité conchylicole) et les artisans, pour des raisons voisines. Il est donc possible d'interpréter de manière fine les positions et oppositions de chacun des groupes dans une situation concrète donnée.

On passe aux préférences des communes en pondérant les coefficients de préférence des agents par le poids que ces agents occu- pent dans la population de la commune (voir tableau en annexe 2). On normalise alors ces préférences communales pour tenir compte de leur intensité et leur direction défavorable à la politique considérée (la composante considérée prend alors une valeur nulle dans le vecteur décrivant le point idéal) ou favorable (la composante prend alors la valeur 1).

On peut finalement considérer les préférences de chaque EPCI en prenant la valeur moyenne des points idéaux (définie sur $\{0,1\})$ et la valeur moyenne des intensités des préférences des communes qui le composent (voir tableau 2), sans les pondérer. Ne pas pondérer ainsi les valeurs exprimant les positions des communes revient à attribuer un même pouvoir de vote à chaque commune. La taille ou la richesse des communes ne sont donc pas considérées comme des critères de pondération pertinents.?

7. Si un règlement interne de l'EPCI impose une répartition spécifique des votes, une pondération cohérente serait retenue. La probabilité que la proposition i soit sélectionnée, wi, serait donc redistribuée en accord avec cette règle. 


\section{Résultats des simulations pour différentes configurations de négociations}

1. Comparaison des accords obtenus pour différentes configurations de négociations

Disposant des estimateurs permettant de caractériser les acteurs, nous pouvons illustrer l'utilisation de l'instrument de simulation. La situation est celle d'un gestionnaire de territoire, animateur et non décideur, qui doit obtenir un accord de la part des décideurs locaux sur les priorités à donner à différents volets des politiques d'aménagement :

- favoriser la croissance urbaine (CU),

- favoriser le tourisme (CT),

- améliorer l'équipement institutionnel et commercial (EQ),

- améliorer l'assainissement (AS).

S'interrogeant sur la stabilité d'un accord obtenu entre EPCI, le gestionnaire cherche à mesurer la sensibilité de cet accord à la structure de négociation retenue, le conduisant à le comparer au résultat de trois situations : - négociation directe entre communes ; - négociation entre EPCI dont le point idéal serait le résultat d'une négociation préalable entre communes membres (dite PIcontrainte pour « contrainte par le point idéal ») ;

- résultat d'une variante de cette dernière configuration dans laquelle la désutilité de l'accord résultant de cette négociation préalable doit être minimisée : toute proposition qui ne réduirait pas cette désutilité pour les communes de l'EPCI, conduirait à son rejet (dite PM-contrainte pour « contrainte par le point de menace »).

Pour ces diverses situations, les solutions obtenues sont présentées dans le tableau 3. Un résultat proche de 0 indique un accord en défaveur de la politique correspondante tandis qu'un résultat proche de 1 indique un accord en faveur de la politique.

Les résultats du tableau 3 montrent qu'une négociation directe entre les 21 communes conduit à un « accord mou » où toutes les orientations sont représentées avec des intensités similaires, de l'ordre de 0,50. La croissance touristique bénéficie d'un léger avantage favorable $(0,56)$. Viennent ensuite l'assainissement $(0,5)$, la croissance urbaine $(0,48)$ et l'équipement $(0,47)$. En revanche, l'issue d'une négociation directe entre EPCI conduit à une valorisation forte de la croissance urbaine $(0,80)$. Les autres dimensions se hiérarchisent comme suit : l'assainissement $(0,56)$, la croissance touristique $(0.51)$ et l'équipement $(0,45)$.

Si les EPCI entrent dans la négociation sur la base des accords passés au sein de chaque communauté (accord PI-contrainte), on retrouve la hiérarchie proposée par les communes seules, avec un engagement un

Tableau 3. Solutions négociées en fonction des configurations de négociations

\begin{tabular}{r|c|c|c|c}
\hline Configurations de négociations & CU & CT & EQ & AS \\
\hline Entre communes seules & 0,4819 & 0,5577 & 0,4682 & 0,4995 \\
\cline { 2 - 5 } Entre EPCI & 0,8023 & 0,5085 & 0,4505 & 0,5614 \\
\cline { 2 - 5 } Entre EPCI (PI-contrainte) & 0,5146 & 0,5959 & 0,4365 & 0,5446 \\
\cline { 2 - 5 } Entre EPCI (PM- contrainte) & 0,6798 & 0,6450 & 0,5215 & 0,6389 \\
\hline
\end{tabular}

Tableau 4. Les accords entre communes au sein de chaque EPCI

\begin{tabular}{rr|c|c|c|c}
\hline Accord & CU & CT & EQ & AS \\
\hline HCB & 0,7576 & 0,7353 & 0,0000 & 0,7278 \\
\cline { 3 - 5 } & 0,6463 & 0,2065 & 0,7348 & 0,4254 \\
\cline { 3 - 5 } & CABT & 0,7888 & 0,6745 & 0,6477 \\
\cline { 3 - 5 } & 0,1150 & 0,6967 & 0,3925 & 0,3575 \\
\cline { 3 - 5 } & CAHM & 0,4426 & \multicolumn{2}{|c}{. }
\end{tabular}


peu supérieur sur le tourisme $(0,60)$, sur l'assainissement $(0,54)$, sur la croissance urbaine $(0,51)$. Les accords locaux renforcent donc (modérément) l'orientation touristique, priorité des accords passés au sein de 3 des 4 EPCI (tableau 4).

L'approche «PM-contrainte » qui favorise la compensation des pertes induites par la recherche d'un compromis valorise les différentes politiques de manière assez semblable : les politiques de croissance urbaine $(0,68)$, du tourisme $(0,65)$ et de l'assainissement $(0,64)$ ont pratiquement le même poids, l'équipement étant d'une ampleur plus faible $(0,52)$.

\section{Graphique 1. Intensité des différentes politiques en fonction des structures de négociations}

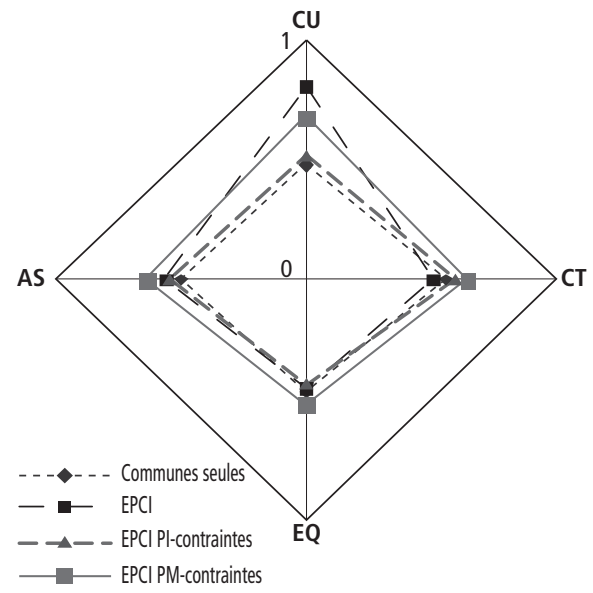

Source : simulation des auteurs

On observe donc un socle de priorités assez voisin pour toutes les configurations de négociation. Ce socle correspond à une politique peu différentiée donnant des poids proches aux quatre orientations possibles. $\mathrm{Ce}$ résultat tend à se déformer lorsque la structure de la négociation favorise les échelons les plus agrégés, c'est-à-dire entre EPCI. La croissance urbaine est alors fortement promue. On observe que donner une garantie aux communes (PM-contrainte) conduit à un accord dont le contenu reste proche de celui auquel conduit une structure de négociation concentrée. Inversement, garantir la satisfaction des accords « locaux » (PI-contrainte) conduit à se rapprocher d'un accord direct entre communes. Autrement dit, plus les objectifs des communes sont respectés (à la différence d'une garantie de compensation des efforts), moins la politique de croissance urbaine est valorisée mais aussi moins est tranchée la politique à suivre.

\section{Comparaison de la stabilité des accords}

De l'analyse du contenu des accords sous divers modes de représentation des parties, il se dégage donc une dynamique liée au choix de la structure de la négociation. La caractérisation de cette dynamique ne permet pas seule la sélection d'une structure plus favorable aux parties prenantes et, pour cette raison, plus acceptable donc plus stable. Nous introduisons donc un critère sur la désutilité relative subie par les communes dans les différentes configurations de négociation. Nous mesurons enfin la moyenne et la variance du rapport des désutilités de chaque configuration à la désutilité des deux cas polaires de notre étude : l'accord décentralisé entre communes et l'accord centralisé entre les $3+1$ EPCI.

L'observation des boîtes de dispersion (graphique 2) montre que chaque configuration est très dispersée pour l'autre pôle, avec des modifications contrastées des désutilités : la négociation entre EPCI est avantageuse pour 9 communes (le ratio désutilité de l'accord entre EPCI/désutilité de l'accord obtenu entre communes y est inférieur à 1) et, inversement, la négociation décentralisée est très favorable à 7 autres communes. Il y a donc une source de tensions potentielles vraie.

L'expression d'intérêts divergents apparaît aussi mais affaiblie, lorsque la décision de l'EPCI doit respecter le montant de perte accepté par les communes (cas 4: PMcontrainte), localement, au sein de chaque EPCI. Enfin la structure de négociation imposant aux communautés l'accord conclu 
RECHERCHES

Patrick RIO, Sophie THOYER

Graphique 2. Coût relatif à supporter par les communes relativement à une structure de négociation donnée [(a) : entre communes; (b) : entre EPCI]

(a)

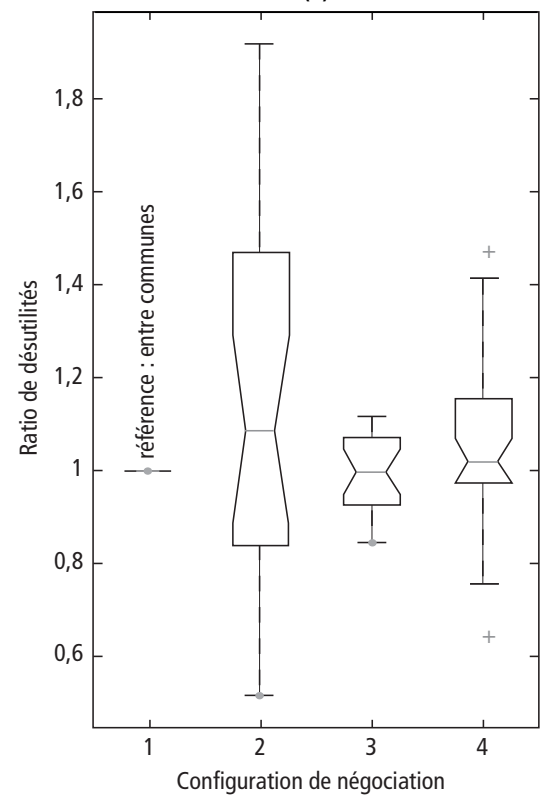

(b)

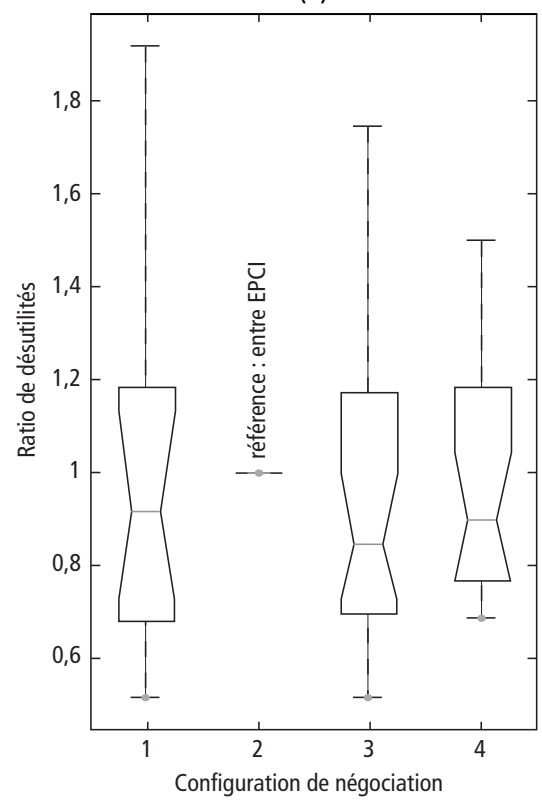

Abscisse : 1 - Communes seules, 2 - EPCI, 3 - EPCI (PI-contrainte), 4 - EPCI (PM-contrainte)

Tableau 5. Désutilités relatives en fonction de la structure de négociation

\begin{tabular}{l|c|c|c|c}
\hline \multicolumn{1}{c|}{$\begin{array}{c}\text { Structure de } \\
\text { négociation }\end{array}$} & \multicolumn{2}{c|}{$\begin{array}{c}\text { Par rapport à une politique } \\
\text { décentralisée }\end{array}$} & \multicolumn{2}{c}{$\begin{array}{c}\text { Par rapport à une politique } \\
\text { centralisée }\end{array}$} \\
\hline & moyenne & variance & moyenne & variance \\
\hline Communes & 1.0000 & 0.0000 & 0.9927 & 0.1356 \\
\hline Communauté & 1.1450 & 0.1626 & 1.0000 & 0.0000 \\
\hline PI-contraintes & 0.9973 & 0.0062 & 0.9773 & 0.1056 \\
\hline PM-contraintes & 1.0582 & 0.0414 & 0.9982 & 0.0700 \\
\hline
\end{tabular}

localement comme point idéal (PIcontrainte) répond de manière opposée à l'une et l'autre politique de référence retenue.

Les valeurs numériques (tableau 5) confirment l'analyse : la structure centralisée avec contrôle communal sur la désutilité maximale (PM-contrainte) représente un meilleur compromis en moyenne (le ratio s'écarte de $5,82 \%$ de la solution décentralisée et de $-0,18 \%$ de la solution centralisée) ; elle compte moins de situations extrêmes parmi les membres (variance de $4,14 \%$ et de $7 \%$ respectivement).
Finalement, ayant déroulé l'information fournie par cet outil d'aide à la décision, le gestionnaire informé des tensions possibles est en mesure de proposer une configuration satisfaisante, en deux étapes :

- favorisant au sein de chaque EPCI l'expression d'un mandat explicite,

- mandat explicite ne portant pas sur l'objectif à défendre mais sur les compensations acceptables dans la recherche d'un compromis.

Il sait que la politique qui en résultera sera peu tranchée, avec une certaine priorité de la dimension «croissance urbaine », 
moindre que l'engagement qui prévaudrait si les EPCI décidaient entre eux. Mais c'est le prix à payer pour une diversité très marquée des intérêts des communes concernées et pour la durabilité d'une politique d'aménagement.

\section{Conclusion}

Cet article présente donc un outil de simulation - couplé à une méthodologie d'évaluation des préférences des communes qui est à la fois relativement simple à mettre en œuvre et très malléable. On peut en effet adapter facilement le modèle de marchandage pour refléter de façon plus précise le poids politique relatif des parties prenantes, pour inclure d'autres acteurs de la négociation ayant des formes d'utilité différentes (par exemple les représentants des usagers) ou pour construire d'autres règles de décision que celle de l'unanimité. C'est donc un outil applicable à de nombreuses configurations de négociation, particulièrement pertinent dans le domaine de l'eau et de l'aménagement du territoire où la participation des communes et EPCI est prépondérante.

Les simulations décrites dans cet article sont donc utiles à la structure porteuse d'un projet de planification concertée. Elles lui permettent d'anticiper la déformation des solutions de compromis en fonction de la façon dont sont structurées les négociations. Elles fournissent un indicateur de stabilité d'un accord passé entre intercommunalités par rapport à un accord passé entre communes. Dans notre exemple, nous avons aussi illustré comment les formes de délégation de pouvoir de négociation des communes à leur intercommunalité peuvent affecter leurs intérêts.
Certes les résultats obtenus ne sont pas directement transposables à la réalité et il ne s'agit pas ici de valider un modèle en le confrontant à la réalité mais plutôt de tester sa faisabilité et d'illustrer les éclairages qu'il peut apporter. Dans le cas de l'étang de Thau, notre outil de simulation nous fournit deux éléments de réflexion intéressants, utiles aux autorités publiques.

- La première réflexion concerne la structuration des négociations en EPCI qui peut avoir tendance à renforcer la priorité donnée aux activités de développement touristique, en synergie avec le renforcement des mesures curatives (investissements dans la modernisation des stations d'épuration) au détriment possible de solutions plus durables fondées sur un développement moins rapide et plus respectueux des exigences écologiques des milieux lagunaires. Ce résultat est dû au renforcement du poids des communes littorales dans la négociation par le jeu des alliances sous-jacentes à la restructuration en communautés de communes et d'agglomération.

- La seconde réflexion concerne le regroupement en EPCI est plus avantageux pour certaines communes que pour d'autres lorsqu'il s'agit de défendre leurs préférences dans une négociation territoriale plus large. Cela peut expliquer la réticence de certaines communes à s'engager dans une logique de regroupement. Dans la perspective d'une fusion entre l'agglomération de Montpellier et les EPCI du bassin de Thau, âprement discutée et aujourd'hui au point mort, ce travail peut fournir un autre éclairage sur les divergences et convergences d'intérêt des acteurs représentés au-delà des simples luttes de partis. 


\section{RÉFÉRENCES BIBLIOGRAPHIQUES}

Adams G., Rausser G., Simon L. (1996). Modeling multilateral negotiations: an application to California water policy, Journal of Economic Behaviour and Organisation, vol. 30, p. 97-111.

Barraque B. (1997). Subsidiarité et politique de l'eau. In Faure A. (éd.), « Territoires et subsidiarité. L'action publique locale à la lumière d'un principe controversé », Paris, L'Harmattan, coll. Logiques politiques, p. 165-201.

Beuret J.-E. (2006). La conduite de la concertation: pour la gestion de l'environnement et le partage des ressources. Paris, L'Harmattan, $340 \mathrm{p}$.

Claeys-Mekdade C. (2003). Le lien politique à l'épreuve de l'environnement : expériences camarguaises. Presses Interuniversitaires Européennes, 245 p.

Comité Régional du Tourisme - Languedoc Roussillon (2002). Les chiffres du camping 1990-1998-2000 Fédération de l'hôtellerie de plein air. http ://www.languedocroussillon.prefgouv.fr/actions/mission littoral/ref_bibliographiques.shtm

Dinar A., Albia J., Sanchez-Soriano (éds.) (2008). Game theory and policy-making in natural resources and the environment, Routledge, 345 p.

Ferroudji-Richard A. (2008). L'appropriation des dispositifs de gestion locale et participative de l'eau. Composer avec une pluralité de valeurs, d'objectifs et d'attachements. Montpellier, Thèse CemagrefEHESS, UMR G-eau, $300 \mathrm{p}$.

Gaudin J.-P. (éd.) (1996). La négociation des politiques contractuelles. Paris, L'Harmattan, $219 \mathrm{p}$.

Insee (1999). Recensement Général de la Population. http://www.recensement1999. insee.fr/RP99/rp99/page_accueil.paccueil

Keeney R.-L., Raiffa H. (1976). Decisions with Multiple Objectives: Preferences and Value Tradeoffs. John Wiley \& Sons, Inc.
Mustajoki J., Hämäläinen R.-P., Marttunen M. (2004). Participatory multicriteria decision analysis with Web-HIPRE: a case of lake regulation policy. Environmental Modelling \& Software, 19-6, June, p. 537-547.

Quérou N., Rio P., Tidball M. (2006). MultiParty Negotiation When Agents Have Subjective Estimates of Bargaining Powers. Group decision and negotiation, on-line first.

Rausser G., Freebairn J.-W. (1974). Estimation of policy preference functions: an application to U.S. beef import quotas. Review of Economics and Statistics, vol. 56, p. 437-449.

Rey-Valette H., Valette F. (2005). Contribution à la définition d'une politique de gestion intégrée de la zone de Thau, étude prospective des scénarios. WP7, études d'appui à la définition des scénarios, Rapport DITTY, $112 \mathrm{p}$.

Richard A. (2000). Analyse comparée de l'acceptabilité des contrats de milieu et des SAGE. Rapport de stage Cemagref, série Irrigation R\&E 2000-06, 55 p.

Rio P., Thoyer S. (2006). Gestion de lagune participative et concertée. Report for the European Project DITTY, Development of an Information Technology Tool for the Management of European Southern Lagoons under the influence of river-basin runoff, $49 \mathrm{p}$.

Saaty T.-L. (1990). Multicriteria Decision Making: The Analytic Hierarchy Process. Pittsburgh, RWS Publications, PA.

Thoyer S, Morardet S., Rio P., Simon L., Goodhue R., Rausser G. (2001). A bargaining model to simulate negotiations between water users. Journal of Artificial Societies and Social Simulation, vol. 4, n 2, http://jasss.soc.surrey.ac.uk/4/2/6.html 


\section{Encadré}

\section{Eléments de discussion sur la démarche mise en œuvre}

Pour plus de précision, nous rassemblons ici quelques éléments de choix qui conditionnent notre démarche. Ces éléments sont cruciaux dans l'obtention de nos résultats et donc pour l'évaluation de notre outil de simulation des négociations. Cet outil est conçu pour fournir aux gestionnaires de la négociation, ici dans un cadre territorial bien défini, un éclairage sur les effets induits sur l'accord d'une structuration particulière de cette négociation. Comme pour tout modèle, nous nous appuyons sur des hypothèses. Simplificatrices: elles ont l'intérêt d'être énoncées en toute transparence et justifiées. Le modèle ne reproduit pas la réalité. II tente d'en saisir ces traits pertinents qui permettent d'interpréter les différences dans les accords obtenus en fonction des scénarios mis en œuvre.

Dans la situation à deux joueurs, chaque joueur propose, dans l'espace des utilités, son point idéal : $A 1$ et $A 2$ sur la figure $A$. On n'est pas dans une situation donnant-donnant (droite A1-A2). L'avantage (social) de coopérer apparaît décrit par la courbe en pointillé : le gain pour la société $\left(u_{1}+u_{2}\right)$ est plus grand que celui qui résulterait d'un jeu à somme nulle bien qu'individuellement cette solution socialement favorable intègre la contrainte résultant de la demande de l'autre.

La situation avec synergies positives est décrite par la courbe passant par B1 et B2. Du fait de la coopération, il existe une position plus favorable pour chaque joueur que leur demande individuelle (les points B1 et B2 sur la courbe qui décrit les situations d'interaction). On peut donc a priori prendre en compte des effets de complémentarité. Ceci, techniquement, se ramène à un changement du système de repères. Passer de I'origine $O$ à l'origine $O$ ' permet de capturer les gains de la coopération. En fait, sur la courbe de coopération passant par B1 et B2, le gain individuellement et indépendamment perçu (en ces points) par chaque joueur agit comme un point de menace possible pour l'autre joueur. D'où la restriction de l'espace de négociation à B1-O'-B2.

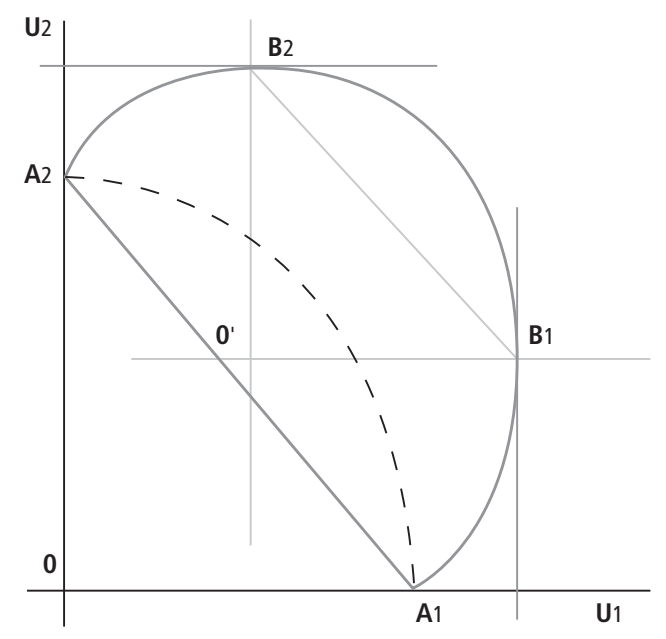

Figure $A$. Négociation avec interactions positives

C'est pourquoi le modèle de marchandage peut capturer les gains tirés d'outils de concertation qui, tels les SAGE ou les SCOT, se proposent de faire évoluer les préférences des parties prenantes vers une meilleure prise en compte de l'intérêt collectif. Comme on le voit dans l'article, un tel modèle de gestion territoriale, de notre point de vue, DOIT coupler un modèle de négociation et, du fait de sa nature d'outil de simulation, un modèle des préférences des acteurs concernés à cet échelon territorial. On utilise ici un modèle économétrique élémentaire de révélation des préférences. Ce n'est pas la seule méthode possible : outre son réel intérêt conceptuel (une théorie du décideur public impliqué dans ce contexte concerté), la nôtre, basée sur les données publiques aisément accessibles de l'INSEE, a un intérêt pratique évident. 


\section{ANNEXES}

\section{Annexe 1}

Le mécanisme est illustré par le graphique Al. À l'étape initiale, les joueurs annoncent leur solution préférée, non contrainte par les demandes des autres joueurs : leur point idéal représenté ici par un losange pour chacun des trois joueurs positionné dans un espace de décision à deux dimensions. Chaque proposition étant énoncée, chaque joueur peut en déduire pour lui-même et pour les autres joueurs l'utilité de s'arrêter à cette étape. Pour cela, chaque joueur va projeter l'utilité espérée de chacun sur l'espace de négociation en déduisant pour chaque valeur d'une dimension, la valeur de la seconde variable induite par l'utilité espérée : le lieu de ces couples $(\mathrm{x} 1, \mathrm{x} 2)$ correspondant dans cet espace à deux dimensions est une courbe comme on le voit au graphique à l'étape 1. Ces courbes, pour un espace compact et des fonctions d'utilité concave, délimiteront un espace borné auquel la solution devra appartenir. Chaque joueur cherchant à minimiser l'écart entre l'utilité de sa demande initiale, son point idéal, et l'utilité d'une proposition admissible (acceptable par les autres joueurs, c'est-à-dire encore une proposition appartenant à l'espace fermé borné défini en étape 1), chaque nouvelle proposition sera sur la frontière de cet espace (étape 2). Mais ainsi, chaque étape conduira à rapprocher les propositions, conduisant simultanément à restreindre l'espace des solutions admissibles comme on le montre avec les étapes 3 et 4 de ce processus : ce mécanisme assure la convergence en temps fini vers un point d'agrément pour tous les joueurs.

\section{Graphique A1. Le processus de marchandage, deux variables négociées, trois joueurs}
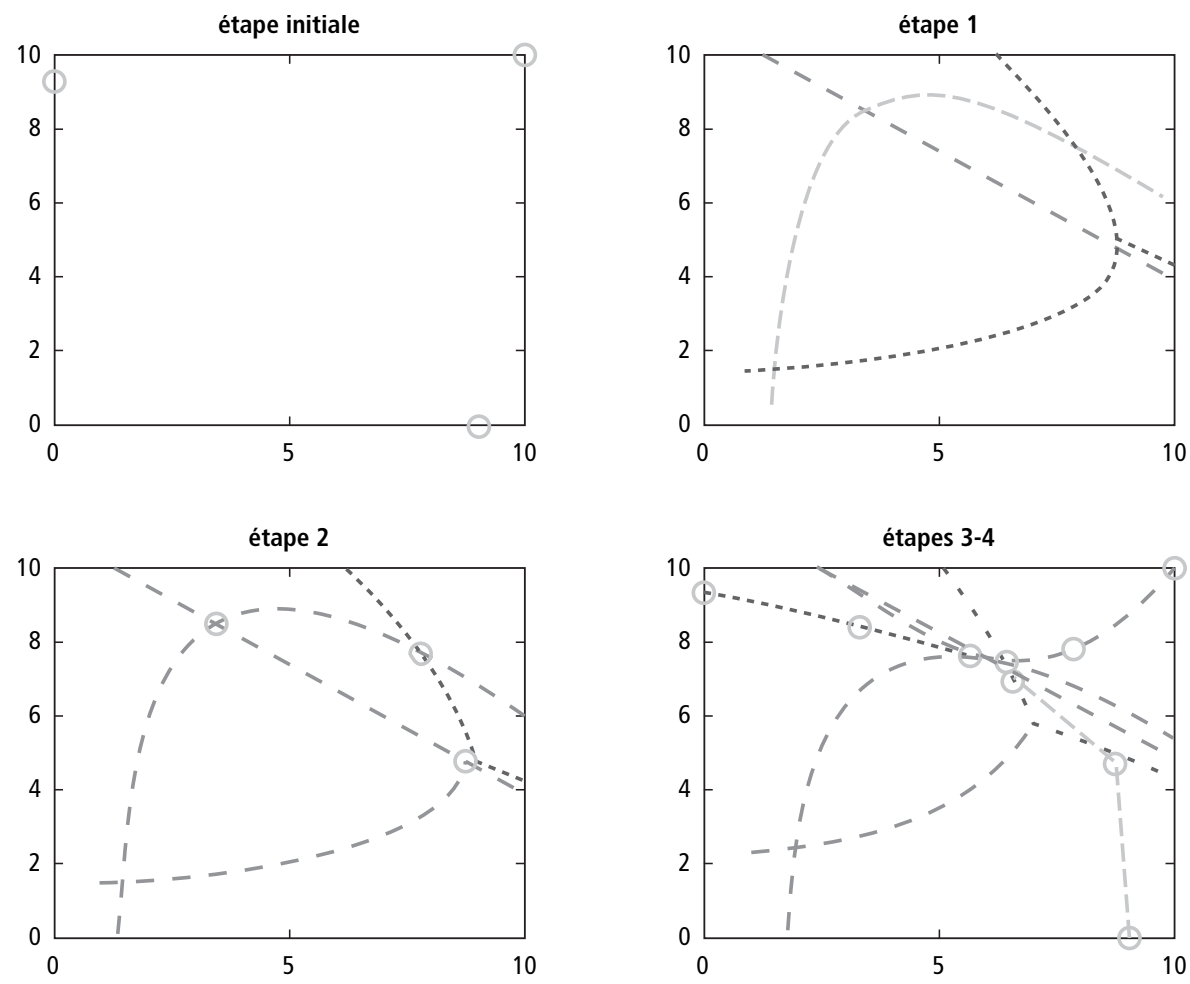


\section{Annexe 2}

Paramètres de préférences des 21 communes du bassin de Thau pour les quatre politiques considérées

\begin{tabular}{|c|c|c|c|c|c|}
\hline Commune $^{(1)}$ & $\begin{array}{c}\text { Croissance } \\
\text { urbaine }\end{array}$ & $\begin{array}{l}\text { Croissance } \\
\text { touristique }\end{array}$ & Équipement & $\begin{array}{l}\text { Assainis- } \\
\text { sement }\end{array}$ & EPCI \\
\hline $\begin{array}{l}\text { Aumelas } \\
\text { Cournonsec } \\
\text { Fabrègues }\end{array}$ & $\begin{array}{l}0,3820 \\
0,4532 \\
0,4728\end{array}$ & $\begin{array}{l}0,0000 \\
0,1374 \\
0,2578\end{array}$ & $\begin{array}{l}0,6065 \\
0,2711 \\
0,2693\end{array}$ & $\begin{array}{l}0,0114 \\
0,1383 \\
0,0000 \\
\end{array}$ & $\begin{array}{c}\text { HCB } \\
\text { hors d'un EPCl du } \\
\text { bassin de Thau }\end{array}$ \\
\hline $\begin{array}{l}\text { Balaruc-le-Vieux } \\
\text { Balaruc-les-Bains } \\
\text { Frontignan } \\
\text { Gigean } \\
\text { Marseillan } \\
\text { Sète }\end{array}$ & $\begin{array}{l}0,3323 \\
0,0825 \\
0,2378 \\
0,4099 \\
0,0918 \\
0,0886 \\
\end{array}$ & $\begin{array}{l}0,1909 \\
0,2803 \\
0,2663 \\
0,2107 \\
0,2842 \\
0,6722 \\
\end{array}$ & $\begin{array}{l}0,3573 \\
0,6070 \\
0,3256 \\
0,2034 \\
0,2954 \\
0,1242 \\
\end{array}$ & $\begin{array}{l}0,1196 \\
0,0302 \\
0,1703 \\
0,1760 \\
0,3286 \\
0,1151\end{array}$ & $\begin{array}{c}\text { CABT } \\
\text { Communauté d'agglomération } \\
\text { du bassin de Thau }\end{array}$ \\
\hline $\begin{array}{l}\text { Bouzigues } \\
\text { Loupian } \\
\text { Mèze } \\
\text { Montbazin } \\
\text { Poussan } \\
\text { Villeveyrac }\end{array}$ & $\begin{array}{l}0,3707 \\
0,1444 \\
0,2323 \\
0,1623 \\
0,1931 \\
0,2418\end{array}$ & $\begin{array}{l}0,1005 \\
0,0812 \\
0,2295 \\
0,2707 \\
0,2016 \\
0,2842\end{array}$ & $\begin{array}{l}0,4032 \\
0,2738 \\
0,0911 \\
0,3820 \\
0,4342 \\
0,3075\end{array}$ & $\begin{array}{l}0,1255 \\
0,5006 \\
0,4471 \\
0,1850 \\
0,1711 \\
0,1666 \\
\end{array}$ & $\begin{array}{c}\text { CCNBT } \\
\text { Communauté } \\
\text { de communes du Nord } \\
\text { du bassin de Thau }\end{array}$ \\
\hline $\begin{array}{l}\text { Agde } \\
\text { Castelnau-de-Guers } \\
\text { Florensac } \\
\text { Montagnac } \\
\text { Pinet } \\
\text { Pomerols }\end{array}$ & $\begin{array}{l}0,0657 \\
0,0307 \\
0,4097 \\
0,2474 \\
0,2406 \\
0,0000 \\
\end{array}$ & $\begin{array}{l}0,3013 \\
0,3104 \\
0,5216 \\
0,4668 \\
0,2969 \\
0,3686 \\
\end{array}$ & $\begin{array}{l}0,4760 \\
0,5429 \\
0,0000 \\
0,1692 \\
0,3332 \\
0,4477 \\
\end{array}$ & $\begin{array}{l}0,1570 \\
0,1160 \\
0,0687 \\
0,1165 \\
0,1294 \\
0,1837 \\
\end{array}$ & $\begin{array}{c}\text { CABT } \\
\text { Communauté } \\
\text { d'agglomération } \\
\text { Hérault Méditerranée }\end{array}$ \\
\hline
\end{tabular}

(1) Seules sont indiquées les communes ayant une partie ou la totalité de leur territoire dans le bassin-versant de la lagune de Thau. 